\title{
Study on two stage supply chain negotiation rescheduling model and algorithm based on non-complete-trust
}

\author{
Hongguang Bo ${ }^{1, a}$, Huanzhi $\mathrm{Li}^{2, \mathrm{~b}}$, Huilin Zhang ${ }^{3, \mathrm{c}}$, Wei Mu ${ }^{4, \mathrm{~d}}$, Guobing LYV ${ }^{5, \mathrm{e}}$ \\ ${ }^{1}$ Faculty of Management and Economics, Dalian University of Technology, Dalian, 1162024, Chain \\ ${ }^{2}$ Faculty of Management and Economics, Dalian University of Technology, Dalian, 1162024, Chain \\ ${ }^{3}$ Faculty of Management and Economics, Dalian University of Technology, Dalian, 1162024, Chain \\ ${ }^{4}$ CSR Qingdao Sifang Co., Ltd. Qingdao, 266111, China \\ ${ }^{5}$ Beijing Xinli Machinery Co., Ltd., Beijing, 100039, China \\ aemail: hgbo@dlut.edu.cn, bemail:15942457182@163.com, \\ cemail:Iazurey@foxmail.com, demail:1098809481@qq.com,eemail:68684592@163.com
}

Keywords: Rescheduling; Non-complete-trust; PSO; Neighborhood search

\begin{abstract}
Aiming at the problem of the two stage supply chain disruption in the JIT production system, this paper first puts forward a disruption management rescheduling model based on non-complete-trust, with cash compensation negotiation mechanism. The time of delivery and the amount of cash compensation as coordinate transmission information, using feedback - consultation mechanisms to solve the information privatization problem. Second, based on the algorithm combination of particle swarm optimization (PSO) and the neighborhood search mechanism, and with examples to verify the negotiation mechanism proposed in this paper can effectively reduce the cost caused by disruption.
\end{abstract}

\section{Introduction}

The rapid development of social economy and science and technology promotes the integration of upstream and downstream in economic industry. In the supply chain management system, Just-In-Time (JIT) manufacturing system has become more and more attention to enterprises [1].But the application of zero inventory, zero defect, zero fault concept of JIT will inevitably require the close connection between the various parts of the supply chain [2]. This also leads to the bullwhip effect of supply chain disruption. Therefore, when the disruption appeared, each enterprise in supply chain needs to react, which can eliminate or mitigate the influence by the negotiation rescheduling.

Supply chain negotiation scheduling researches on the supplier, manufacturer and transportation enterprises in the process of disruption, how to improve the efficiency of the whole supply chain through the negotiation strategy to achieve the ultimate realization of their own scheduling gains. Chen [3] analyzes of the contradiction and cooperation between the supplier and the manufacturer in the production of assembly supply chain disruption composed of one supplier and several manufacturer. Dan.B [4] uses batch scheduling and scheduling as two sub problems to deal with. Manoj [5] studies negotiation scheduling problem of a supply chain consisting of a manufacturer, a carrier and several retailers. Aytug [6] makes systematic review of the latest progress. However, there is no consideration of the information private problem between the supply chain partners when the disturbance is produced.

Supply chain composed of two enterprises is the most basic supply chain structure, but also is the basis of other supply chain, has a very important position in the supply chain management. On the basis of the above studies, this paper establishes a disruption management model with non-complete-trust among the supply chain enterprises in the two stage, one supplier and one manufacturer, establishing the scheduling scheme and the negotiation cash compensation to solve the information privatization problem in the supply chain. 


\section{Information sharing and trust between enterprises after the disruption}

In the JIT system, there are a lot of invisible information flows, such as the visible raw materials, semi-finished products, products and so on. However, considering that the information itself has the characteristics of non-segmentation and non-exclusive, or one party has a certain control (the buyer's market or the seller's market), and the distance from the end consumer. These led the supply chain enterprises to grasp the information asymmetry, and then the enterprise is not trust or non-complete-trust.

The above mentioned studies have indicated that the information sharing among the members in the supply chain can effectively increase the efficiency of the whole supply chain operation and the efficiency of themselves. But in the actual operation process, the degree of information sharing is restricted by the degree of trust.

The enterprises in the supply chain have the contract and other binding requirements for information exchange. But in the actual operation, it is difficult to achieve information sharing and complete trust between enterprises. On the one hand, the information provided to other members inevitably has some distortion. On the other hand, taking into account the privacy of information, and even some of the information are business secrets, the enterprise will not provide accurate information. So in the process of sharing between enterprises cannot be complete-trust. These are the great obstacles to the efficiency improvement, but in reality they often exist.

\section{Description}

This paper considers a supply chain consisting of a supplier $U$ and a manufacturer $\mathrm{D}$, they are two independent enterprises. Normal condition in the supply chain is the supplier $U$ first task scheduling to minimize the maximum completion time, because this production planning can maximize its production line utilization, then provide products to $\mathrm{D}$ for the next stage of production. $\mathrm{D}$ on the basis of adapting to the delivery time to scheduling. In the market economy, time is money, so the manufacturer $\mathrm{D}$ also task scheduling with the objective of minimizing the maximum completion time.

Manufacturer D has a number of customers, but the supply relationship with the supplier $U$ is its lifeblood, because the supplier $\mathrm{U}$ goods quality is the best. $\mathrm{D}$ hope that supplier $\mathrm{U}$ will give priority to meet its orders. In the actual, the disruption events occur, resulting in the original planning task changed, so that the initial optimal scheduling is not optimal or feasible. In order to reduce the cost of disturbance, manufacturer D needs to take the task rescheduling, and according to the optimal rescheduling to arrange new product delivery time, and hope that the supplier $\mathrm{M}$ can adjust the production plan to adapt to this. But it also knows that if the supplier $\mathrm{U}$ acceptance of new delivery times, that means that the supplier's original optimal production plan cannot be implemented, will produce more cost, so there will be a fine compensation. And the information cannot be fully shared, the manufacturer $\mathrm{D}$ does not know the exact timing of the job processing in supplier $\mathrm{U}$, they are non-complete-trust.

\section{Model design}

$\operatorname{Job}_{j}(j=1, \ldots, n)$ in supplier $\mathrm{U}$ and manufacturer $\mathrm{D}$ required processing time are expressed as $p_{j, u}$ and $p_{j, d}$, delivery time is $d_{j}$. Assume that all jobs are released at zero time. That is, the start time of the supplier $U$ to the job is 0 , and the release time of the job in the manufacturer's $D$ is $r_{j}$.Its value depends on it completion time in supplier U. $w_{j}$ is the job in supplier $\mathrm{U}$ unit delay penalty weight per unit cost. $s_{j, u}$ and $e_{j, u}$ respectively indicate start and finish time of the job in the supplier's manufacturing process. $s_{j, d}$ And $e_{j, d}$ respectively indicate the start and finish time of the job in the manufacturer manufacturing process, $y_{j, d}$ indicate the degree of disturbance.

Supplier and manufacturer should be in line with the actual production, which means manufacturer's start production time shall not be earlier than the supplier's completion time, which 
is $s_{j, d} \geq e_{j, u}$. Their objective function is based on the time, so it is needed to use the function $\varepsilon$ to convert the time to cost. Production environment is proportional two-machine.

Design disruption scheduling model:

(1) non-complete-trust and information private

Manufacturer D can only be roughly estimated the job processing time in supplier. Joining this time to itself processing time to the start time and end time calculation, and then subtracting the estimated processing time from, so as to determine the delivery time.

(2) Negotiation compensation model

To encourage supplier $U$ to adopt new scheduling plan, manufacturer $\mathrm{D}$ giving the compensation cash $\sigma$ to supplier, after the manufacturer subtracted the compensation from the new scheduling target value, and the result should be better than the original scheduling [7]. $\alpha, \beta$ express the scheduling plan of supplier $U$ and manufacturer $\mathrm{D}$ in front of the disruption. $\alpha$ And $\beta^{\prime}$ are expressed the new scheduling of supplier $U$ and manufacturer $D$ under the negotiation scheduling model. Expressed as follows:

$$
\begin{aligned}
& \varepsilon\left(\alpha^{\prime}\right)-\delta \leq \varepsilon(\alpha) \\
& \varepsilon\left(\beta^{\prime}\right)-\delta \leq \varepsilon(\beta)
\end{aligned}
$$

\section{Model solution design}

In this paper, we designed the algorithm based on the combination of the basic PSO and the neighborhood search mechanism, to solve the disruption management scheduling model. Specific steps are as follows:

\section{Step 1:}

Input the manufacturer $\mathrm{D}$ job schedule, the job processing is affected by disruption and the time longer; manufacturer $\mathrm{D}$ guess the supplier $\mathrm{U}$ job processing time; calculate the delivery time; schedule according to EDD rule.

\section{Step 2:}

Enter the manufacturer's parameters and $d_{j, u}$, to determine whether there is a delay; in accordance with the SPT rules to resort the work sequence has been obtained; the optimal scheduling problem of manufacturer.

\section{Step 3:}

(1) PSO algorithm phase

Optimization the two stage of before the disruption according to the working principle of under the proportional two-machine production environment. Using the constraint of $s_{j, d} \geq e_{j, u}$ to update processing schedule Timesheet

(2) The handling of disruption and non-complete-trust

In the generation of disruption, using the solution from previous two steps as heuristic solution, rethought the particle swarm optimization algorithm and re-optimization. At the same time, the manufacturer D to predict the processing time of the job in the supplier $U$, after optimization and then subtract the estimated processing time, Step 3 backward projection to get a new Timesheet.

\section{PSO algorithm and neighborhood search strategy design}

Insert neighborhood search strategy: As shown in Figure 2, that is, job $k$ random forward insertion, which job $k_{1}$ is located in the random position.

Swap neighborhood search strategy: as shown in Figure 3, exchange the location of the last job of each particle and the next particle swarm of the first job. 


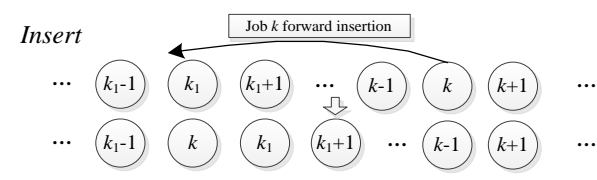

Fig. 2. Insert structure

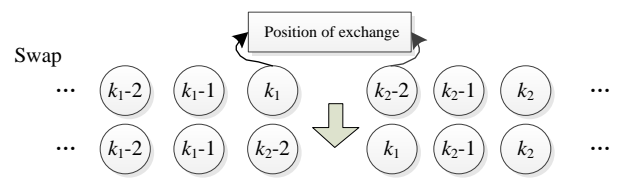

Fig. 3. Swap structure

Considering two kinds of neighborhood search advantage, high efficient of Insert and easy to jump out of local extreme to increase the scope of the search feature of Swap, this paper adopts a hybrid neighborhood structure: Insert-Swap, it is a random selection strategy choose one or both of these neighborhood search way . The random neighborhood structure is defined as: $F\left(c_{P I I} \otimes X_{\text {best }}(g)\right)$, that means the local search for each generation of optimal individual execution step size is $\bar{M}$ on the ${ }^{C_{P I I}}$ probability. ${ }^{C_{P I I}}$ is probability distribution interval for selection: $c_{p i}\left[\alpha_{1}, \beta_{1}\right]$ and $c_{p i i}\left[\alpha_{2}, \beta_{2}\right]$.The corresponding relationship of random neighborhood search operator $F\left(c_{P I I} \otimes X_{\text {best }}(g)\right)$ between the interval of probability distribution and the choice of search operation is shown formula 3:

$$
c_{P I I}=\left\{\begin{array}{cccc}
c_{p i}\left[\alpha_{1}, \beta_{1}\right] & \left(\alpha_{1} \leq \beta_{1}\right) & \Rightarrow & \operatorname{Insert}\left(\pi_{1}, v, \pi_{2}\right) \\
c_{p i i}\left[\alpha_{2}, \beta_{2}\right] & \left(\alpha_{2} \leq \beta_{2}\right) & \Rightarrow & \operatorname{Swap}\left(\pi_{1}, v_{1}, \pi_{2}, v_{2}\right)
\end{array}\right.
$$

Taking into account the special circumstances that may occur, the probability distribution may overlap, so delimiting a combination of neighborhood structure: $C O M<I, S>$, to describe the combination state of two operators, and order the priority sequence of the two operators in the combined state

Particle velocity and position updating by formula 4 :

$$
\begin{gathered}
v[i, j]=\left(\left(0.7 * \frac{M a x-I}{100}\right)+0.5\right) * v[i, j]+c 1 *(p[i, j]-A[i, j])+c 2 *(p[j]-A[i, j]) \\
A[i, j]=A[i, j]+v[i, j]
\end{gathered}
$$

In the type: $A[i, j]$ is particle position coordinates; $v[i, j]$ is particle velocity vector; Max is the total number of iterations; $I$ is the number of iterations at this time; $c 1$ and $c 2$ are constant; $p$ is particle optimal position at this time.

\section{Test results}

In order to verify the validity of the negotiation scheduling model, the paper designs an experiment. Assuming the effect of the disruption is the job processing time expanded from 1.5 to 2.5 random multiple. The manufacturer $\mathrm{D}$ guesses that the processing time of a job in the $\mathrm{U}$ is the product of the number of its own and a random number, the random number range between 1.5 2.5.

Particle swarm size is 50, 200 consecutive iterations, neighborhood step size is set to 10, value of $c 1$ and $c 2$ is 2, the selection probability interval neighborhood structure algorithm for neighborhood operator: $\alpha_{1}=0.0, \alpha_{2}=0.4, \beta_{1}=0.6, \beta_{2}=1.0$. When the current optimal solution cannot be improved, stop iteration. Algorithms are coded C\# language and running in Studio2010 Visual, the operating environment of the algorithm are Core i3-2120CPU @3.30GHz /4G dual core DDR3/ Windows7 32 bit SP1.

When the number of jobs were 10, 20, 50, 70, each example calculating 5 times, and record the convergence generation and the best value (algebraic convergence value). The disruption from their respective job size is 3 randomly selected, the final the results are shown in table 1. 
Table 1 The convergence situation after the disruption

\begin{tabular}{|c|c|c|c|c|c|c|c|}
\hline \multicolumn{2}{|c|}{10} & \multicolumn{2}{c|}{20} & \multicolumn{2}{c|}{50} & \multicolumn{2}{c|}{70} \\
\hline $\begin{array}{c}\text { Convergence } \\
\text { generation }\end{array}$ & best & $\begin{array}{c}\text { Convergence } \\
\text { generation }\end{array}$ & best & $\begin{array}{c}\text { Convergence } \\
\text { generation }\end{array}$ & best & $\begin{array}{c}\text { Convergence } \\
\text { generation }\end{array}$ & \begin{tabular}{c} 
best \\
\hline 35
\end{tabular} \\
\hline 87 & 92.4 & 62 & 92.5 & 36 & 101.9 & 44 & 183.1 \\
\hline 55 & 86.8 & 79 & 126.7 & 41 & 152.0 & 36 & 160.2 \\
\hline 73 & 73.8 & 63 & 97.0 & 40 & 131.6 & 45 & 182.6 \\
\hline 127 & 6.89 & 68 & 117.6 & 46 & 94.6 & 49 & 184.9 \\
\hline
\end{tabular}

It can be seen that, in the mechanism of the compensation negotiation, by combining the PSO algorithm with the neighborhood search algorithm, can get a good stable solution, and ultimately find the lowest cost scheduling.

\section{Conclusion}

In this paper, a study is made on the disruption of a two stage supply chain consisting of one manufacturer and one manufacturer. Using manufacturer's compensation as a negotiation condition and putting forward the negotiation model of supply chain under JIT system. The process optimization strategy based on PSO and neighborhood search mechanism is designed, and the algorithm is proved to be effective.

In the actual production, the environment is more complex. Needing to design more perfect multi-objective evaluation method .These needs our further research in the future.

\section{Acknowledgement}

In this paper, the research was sponsored by the National Science and Technology Support Project of China (Project No. 2015BAF08B02), the Social Science Planning Fund of Liaoning, 2017 Social Sciences Association Fund of Economic and Social Development of Liaoning (Project No. 2017lslktyb-042), National University and Vocational College Logistics Course Reform Fund (Project No.JZW2016027) and Teaching Reform Fund of Dalian University of Technology (Project No.ZD2016011).

\section{References}

[1] LIU Xiao, WANG Cheng-en, CHU Cheng-bin. A JIT Planning Approach for Crude Oil Procurements in Distributed Supply Chain [J]. Chinese Journal of Management Science, 2003, 11(3): 30-35.

[2] Cao Cuizhen. The essence and implementation conditions of JIT mode of production [J]. On Economic Problems, 2004 (11): 46-47.

[3] Chen Z L, Hall N G. Supply chain scheduling: Conflict and cooperation in assembly systems [J]. Operations Research, 2007, 55(6): 1072-1089.

[4] Dan B, Xiao J, Liu X, et al. Batch scheduling of a multi-stage supply chain with due windows[J]. COMPUTER INTEGRATED MANUFACTURING SYSTEMS-BEIJING, 2007, 13(2): 310.

[5] Manoj U V, Gupta J N D, Gupta S K, et al. Supply chain scheduling: Just-in-time environment[J]. Annals of Operations Research, 2008, 161(1): 53-86.

[6] Aytug H, Lawley M A, McKay K, et al. Executing production schedules in the face of uncertainties: A review and some future directions [J]. European Journal of Operational Research, 2005, 161(1): 86-110.

[7] Su S, Yu HJ, Wu ZH, Tang Y. Negotiated scheduling model and algorithms of supply chain. [J].Journal of Software, 2013, 24(1): 12-24. 